\title{
Signal processing techniques for anywhere, anytime positioning
}

\author{
Marco Luise ${ }^{1 *}$, Carles Fernández-Prades ${ }^{2}$, Sinan Gezici ${ }^{3}$ and Henk Wymeersch ${ }^{4}$
}

In the wide and active research community dealing with positioning techniques, the idea of being able to seamlessly locate a device, regardless of its particular location (indoor, outdoor, in the air, at sea, etc.), has been around for decades. More recently, the value of location-based applications in diverse fields such as homeland security and disaster management, transport, logistics and advertising, and tourism, just to mention a few, has also been widely recognized. We are thus witnessing a blooming of different technologies to eventually help coming to the goal of anywhere, anytime positioning $(A A P)$ : the ability of having available at any time the indication of the location of a device anywhere in the world.

We could compare and parallel the requirement of this AAP feature and the development of the necessary technology with what has already happened in the field of wireless communications: ubiquitous cellular networks have now successfully come to their fourth generation with LTE-A technologies that are spreading all over the world, and the fifth generation is now being studied in many R\&D labs. A similar evolution can be seen in satellite-based positioning, where we may label GPS as the first generation and GALILEO and GPS II as the second, with the third generation being developed right now (in Europe, the GALILEO II Generation project).

With respect to wireless communications, positioning is severely lagging behind in terms of standardization efforts: when it comes to terrestrial-based positioning, be it indoor or outdoor, an international agreement on a set of standard techniques to be developed and adopted still has to come. In addition to this, no universal procedure to perform a 'seamless handover' between satellite and terrestrial technologies (or in general between two different positioning techniques) has been defined yet, adding further friction against a smooth development of AAP. Curiously, the sole example of a truly universal location procedure that is going to become a de facto standard is

\footnotetext{
* Correspondence: marco.luise@iet.unipi.it

'CNIT, @University of Pisa, Via G. Caruso 16, Pisa 56126, Italy

Full list of author information is available at the end of the article
}

the one embedded as an ancillary function into the fourth generation of cellular networks through a dedicated ranging channel. Again, no recognized standard approach is going to be used in indoor applications, and a plethora of different and competing technologies with different features and costs is still being developed and proposed (think of RFID-based versus UWB-based location).

This special issue is another testimony to this 'technology spread', featuring a number of high-quality research contributions based on different technologies such as acoustics, sensor networks, inertial devices, opportunity radio signals, etc. It is also a testimony to the many fields in which those technologies can be applied, such as unmanned aerial vehicles (UAVs), transportation systems, groups of persons, and so on. In the first paper of this issue entitled 'Performance evaluation of an acoustic indoor localization system based on a fingerprinting technique, Aloui, Raoof, Bouallegue, Letourneur, and Zaibi present an acoustic location system that adopts the time of arrival of the path of maximum amplitude as a signature and estimates the target position through nonparametric kernel regression, intended for indoor multiuser applications. Chen, Gao, and Wang, in their paper 'Robust all-source positioning of UAVs based on belief propagation', introduce a positioning algorithm named Belief propagation-based Opportunistic Positioning of UAVs (BOPU) with an unscented particle filter for Bayesian approximation. The scenario is that of a group of formation-flying UAVs that use satellite positioning as well as any other wireless signals that are available to increase robustness of their location estimation. The third paper 'Upper bounds on position error of a single location estimate in wireless sensor networks', authored by Gholami, Ström, Wymeersch, and Gezici, studies upper bounds on the position error for a single estimate of an unknown target node position based on distance estimates in a wireless sensor network. The authors formulate in particular their bounds as nonconvex optimization problems and propose a simple approach to find such bounds in closed forms. A particular topic in cooperative localization is tackled by the fourth paper 'Schedule-based sequential localization in 
asynchronous wireless networks' by Zachariah, De Angelis, Dwivedi, and Händel, in which the authors consider a socalled schedule-based network localization concept, which does not require synchronization among nodes and does not involve communication overhead. Their concept makes use of a common transmission sequence, which enables each node to perform self-localization and to localize the entire network, based on noisy propagation-time measurements. Zachariah and Händel also authored the subsequent paper, together with Nilsson and Skog, that is entitled 'Cooperative localization by dual foot-mounted inertial sensors and inter-agent ranging' and describes the (partially decentralized) architecture of a whole positioning system for a platoon-sized group of agents featuring dual foot-mounted inertial sensors and based on inter-agent ranging. Such an architecture is believed to greatly reduce the computational cost and required communication bandwidth while only giving negligible information loss in comparison with a conventional centralized implementation. Del Peral-Rosado, López-Salcedo, Seco-Granados, Zanier, and Crisci, in their paper 'Joint maximum likelihood time-delay estimation for LTE positioning in multipath channels', present a joint time-delay and channel estimator to assess the achievable positioning performance of the LTE networks, whose performance is heavily affected by radio multipath. They show that LTE pilot signals can be used to estimate the multipath channel and thus improve time-delay estimation with respect to conventional correlation-based estimators. The paper 'Adaptive mobile tracking in unknown non-line-of-sight conditions with application to digital TV networks' authored by L. Chen, Piché, Kuusniemi, and R. Chen analyzes the issue of achieving position information through the use of signals of opportunity from a digital TV (DTV) network. They propose to use an adaptive Rao-Blackwellized particle filter (RBPF) for mobile tracking in such scenarios, with an extended Kalman filter to estimate the mobile state. The particle filter is thus applied to estimate the posterior distribution of sight conditions and the unknown static parameters. An interesting approach to the general issue of target tracking is described in the subsequent paper 'Tracking target signal strengths on a grid using sparsity' by Farahmand, Giannakis, Leus, and Tian, whose main feature is the introduction of a grid-based model in which the state captures target signal strengths on a known spatial grid (TSSG). This model leads to linear state and measurement equations, which bypass data association and is amenable to state estimation via sparsity-aware Kalman filtering (KF). The novel TSSG trackers do not require knowing the number of targets or their signal strengths and exhibit considerably lower complexity than the benchmark hidden Markov model filter, especially for a large number of targets. In the ninth paper entitled 'Source positioning in a large-scale tiny-sensor network of arbitrary topology', the authors Penkin, Janssen, and Yarovoy investigate the special problem of locating a source node in a dense wireless tiny sensor network. In their approach, the network topology is described as a regular lattice, and the statistical parameters of the hop count in the source-to-sink propagation path are related to the fractal properties of a percolating cluster. Based on this approach, a mathematical model is developed to estimate the probability of successful reception of the message by a sink node spaced at a certain distance from the source in a given time interval. The final contribution to this special issue is the paper by Moon, Chun, Hur, and Jee entitled 'A T-DMB Navigation System for Seamless Positioning both in Indoor and Outdoor Environments'. The authors describe how to perform positioning of a receiver using a single-frequency synchronized network like the one deployed in many countries for terrestrial digital television broadcasting. The mobile user measures a time difference of arrival (TDOA) for all $\mathrm{T}$ DMB transmitter pairs available in a certain area. The time difference is converted into a distance difference, and so the mobile user position can be estimated. Experimental results from the Seoul area show that the measurement error is bounded under $300 \mathrm{~m}$ and can be used to determine the mobile user's position with a small standard deviation.

We wish to acknowledge here the support to the preparation of this special issue that came from the Network of Excellence in Wireless Communications NEWCOM\#, funded under the umbrella of the 7th Framework Program of the European Commission (FP7-ICT-318306). We also sincerely thank all of the researchers that contributed to this special issue, and we do hope that the collection of results they provided may contribute to the advancement of the state of the art in the field of positioning, as well as encourage young researchers to work in this fast-developing and exciting area.

\section{Author details}

${ }^{1}$ CNIT, @University of Pisa, Via G. Caruso 16, Pisa 56126, Italy. ${ }^{2} \mathrm{CTTC}$, Av. Carl Friedrich Gauss, 7, Castelldefels 08860, Spain. ${ }^{3}$ Bilkent University, Üniversiteler Mh., Ankara 06800, Turkey. ${ }^{4}$ Chalmers University of Technology, SE-41296 Göteborg, Sweden.

Received: 5 June 2014 Accepted: 5 June 2014 Published: 17 June 2014

doi:10.1186/1687-6180-2014-93

Cite this article as: Luise et al:: Signal processing techniques for anywhere, anytime positioning. EURASIP Journal on Advances in Signal Processing 2014 2014:93. 